\title{
The plastid genome characteristics of a moth orchid (Phalaenopsis wilsoniii, Orchidaceae)
}

\author{
KeKe Xia ${ }^{1}$, Ding-Kun Liu ${ }^{2}$, Jie-Yu Wang ${ }^{3,4 *}$ \\ ${ }^{1}$ BGI-Shenzhen, Shenzhen 518083, China \\ ${ }^{2}$ Key Laboratory of National Forestry and Grassland Administration for Orchid Conservation and \\ Utilization at Colleage of Landscape Architecture, Fujian Agriculture and Forestry University, \\ Fuzhou 350002, China \\ ${ }^{3}$ Key Laboratory of Plant Resources Conservation and Sustainable Utilization, South China Botanical \\ Garden, Chinese Academy of Sciences, Guangzhou 510650, China \\ ${ }^{4}$ Laboratory for Orchid Conservation and Utilization, The Orchid Conservation and Research Center \\ of Shenzhen, The National Orchid Conservation Center of China, Shenzhen 518114, China
}

${ }^{*}$ Corresponding authors.

E-mail addresses: jywang@scbg.ac.cn (J.-Y. Wang) 


\author{
Abstract \\ Phalaenopsis wilsonii is a typical deciduous species in the horticulturally well-known genus, Phalaenopsis. Tshi \\ species is belonging to the section Aphyllae in moth orchid, and is endemic to South China. Although the Aphyllae \\ section display the deciduous feature that is unique in this genus, their genetic information is still insufficient and \\ limited them as breeding parent in moth orchid. Here, we reported and characterized the complete chloroplast \\ genome for Phalaenopsis wilsonii. We found the total size of the chloroplast genome was 145,373 bp, constituting \\ of a large single copy (LSC) region (84,996 bp), a small single-copy region (10,668 bp) and two \\ inverted repeats (IRs) regions $(24,855 \mathrm{bp})$. Based on homologous searching on database, we annotated 76 protein- \\ coding genes, 38 tRNA, and 8 rRNA. The phylogenetic reconstruction revealed that $P$. wilsonii show the closest \\ relationship with $P$. lowii within subgenus Parishianae.
}




\section{Introduction}

Moth orchid (Phalaenopsis Blume) is widely used in gardening around the world, and also occupied a larger proportion of orchid industry (Van Huylenbroeck 2018). Recently, the taxonomy of Phalaenopsis reached a congruence in perspectives of morphological and molecular evidence, resulting in four subgenera, subgen. Phalaenopsis, Parishianae, Hygrochilus and Ornithochilus (Kocyan and Schuiteman 2014; Li et al., 2014, 2016). Subgen. Parishianae consists of more than a half of the species richness in Phalaenopsis, and is also an important breeding resource, however, the genetic data of this section is quite insufficient. So far, only one complete chloroplast genome of species belonging to this subgenus can be found (Wang et al., 2019), and no transcriptome or nuclear genome data have been published. $P$. wilsonii is endemic in China, and is also a typical deciduous Phalaenopsis that is belonging to subgen. Parishianae. Here, we provide a complete chloroplast genome of $P$. wilsonii, which will facilitate our understanding on Phalaenopsis breeding and future designment of molecular markers.

\section{Methods and materials}

The sampling individual of Phalaenopsis wilsonii is cultivated in National Orchid Conservation Centre in

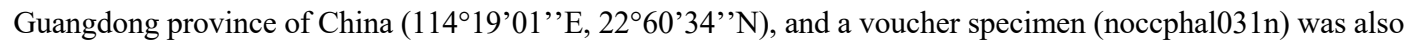
deposited in the Herbarium of National Orchid Conservation Centre, Shenzhen, China. A young leaf from the voucher specimen was sampled to extract the total DNA, and sequencing was performed by Illumina HiSeq 2000 platform (Illumina, San Diego, CA). To obtain the chloroplast source reads, we reconstruct the reference database based on all published Phalaenopsis plastid genomes, and mapped our clean reads against them to obtain the chloroplast reads for $P$. wilsonii. We used PLATANUS (Kajitani et al., 2014) to construct the initial contigs, and linked the contigs based on the same reads for the scaffolds, resulting in the final complete genome after artificial modification. The BLAST was used to map the reads onto the genome again to confirm the IR boundaries, and the annotation was performed using Geneious 2019.0.3. The resulting complete chloroplast genome of $P$. wilsonii was submitted to GenBank under the accession number of MW218959. The syntenic block detecting and maximumlikelihood phylogenetic reconstruction are conducted by Homblock (Bi et al., 2018) and RAxML, respectively. The matrix contained the whole plastid genomes of $P$. wilsonii, four moth orchids, and also the other ten orchids. Cattleya crispate was applied as outgroup according to the topology from Givnish et al. (2015). 
The total length of $P$. wilsonii chloroplast genome is $145,373 \mathrm{bp}$, which is slightly less than other published moth orchids (from $146,834 \mathrm{bp}$ in $P$. lowii to $148,964 \mathrm{bp}$ in P. aphrodite subsp. formosana) with the GC content as $36.9 \%$. As in other orchids, the chloroplast genome of $P$. wilsonii consists of a large single copy (LSC) region (84,995 bp) and a small single-copy region (10,668 bp), which were separated by two inverted repeat (IRs) regions (24,855 bp). Overall, 122 genes (contain repeat region gene) were annotated, including 76 protein-coding genes, 8 rRNAs, and 38 tRNAs. As similar to the description in (Chang et al., 2005), all $n d h$ genes of $P$. wilsonii were also non-functional, and the $n d h E$ was also lost.

Our phylogenetic tree showed a similar topology to Givnish et al. (2015), where the Vandeae presented a sister relationship to Cymbidieae. And P. wilsonii was sister to P. lowii, and these two species belonging to subgen. Parishianae formed the single clade (Figure 1). This complete chloroplast genome of $P$. wilsonii will be the useful information for future phylogenetic studies and conservation in Phalaenopsis.

\section{Acknowledgement}

This work was supported by the Science, Technology and Innovation Commission of Shenzhen Municipality under Grant No. JCYJ20170817151501595.

\section{Authors' contributions}

J.Y.W. and K.K.X. conceived the study; J.Y.W. and K.K.X. obtained the molecular data; J.Y.W. and D.K.L. conducted the data analysis; K.K.X. drafted the manuscript; J.Y.W. and D.K.L. revised the manuscript. All authors provided comments and final approval.

\section{Data Availability Statement}

The data that support the findings of this study are openly available in GenBank of NCBI at https://www.ncbi.nlm.nih.gov, reference number MW218959.

\section{References}

Bi, G, Mao, Y, Xing, Q, et al., 2018. HomBlocks: a multiple-alignment construction pipeline for organelle phylogenomics based on locally collinear block searching. Genomics 110 (1): 18-22.

Chang, C.-C., Lin, H.-C, Lin, I.-P., et al., 2005. The chloroplast genome of Phalaenopsis aphrodite (Orchidaceae): comparative analysis of evolutionary rate with that of grasses and its phylogenetic implications. Molecular Biology and Evolution 23 (2): 279-291. 
Givnish, T.J., Spalink, D., Ames, M., et al., 2015. Orchid Phylogenomics and Multiple Drivers of Their Extraordinary Diversification. Proceedings of the Royal Society B: Biological Sciences 282 (1814): 20151553. doi:10.1098/rspb.2015.1553.

Kajitani, R, Toshimoto, K, Noguchi, H, et al., 2014. Efficient de novo assembly of highly heterozygous genomes from whole-genome shotgun short reads. Genome Res. 24 (8):1384-95.

Kocyan, A., and Schuiteman, A., 2014. New combinations in Aeridinae (Orchidaceae). Phytotaxa 161 (1): 61-85.

Li, M.-H., Gruss, O., and Liu, Z.-J., 2016. Nomenclature changes in Phalaenopsis subgen. Hygrochilus (Orchidaceae; Epidendroideae; Vandeae) based on DNA evidence. Phytotaxa 275 (1): 55-61.

Li, M.-H., Zhang, G.-Q., Liu, Z.-J., and Lan, S.-R., 2014. Revision of Hygrochilus (Orchidaceae: Epidendroideae: Aeridinae) and a molecular phylogenetic analysis. Phytotaxa 159 (4): 256. doi:10.11646/phytotaxa.159.4.2.

Van Huylenbroeck, J.. 2018. Ornamental Crops. Springer.

Wang, J.-Y., Liu, Z.-J., Zhang, G.-Q., and Peng, C.-C., 2019. The Complete Chloroplast Genome Sequence of Phalaenopsis Lowii (Orchidaceae). Mitochondrial DNA Part B 4 (2): 3569-3570.

doi:10.1080/23802359.2019.1674715. 


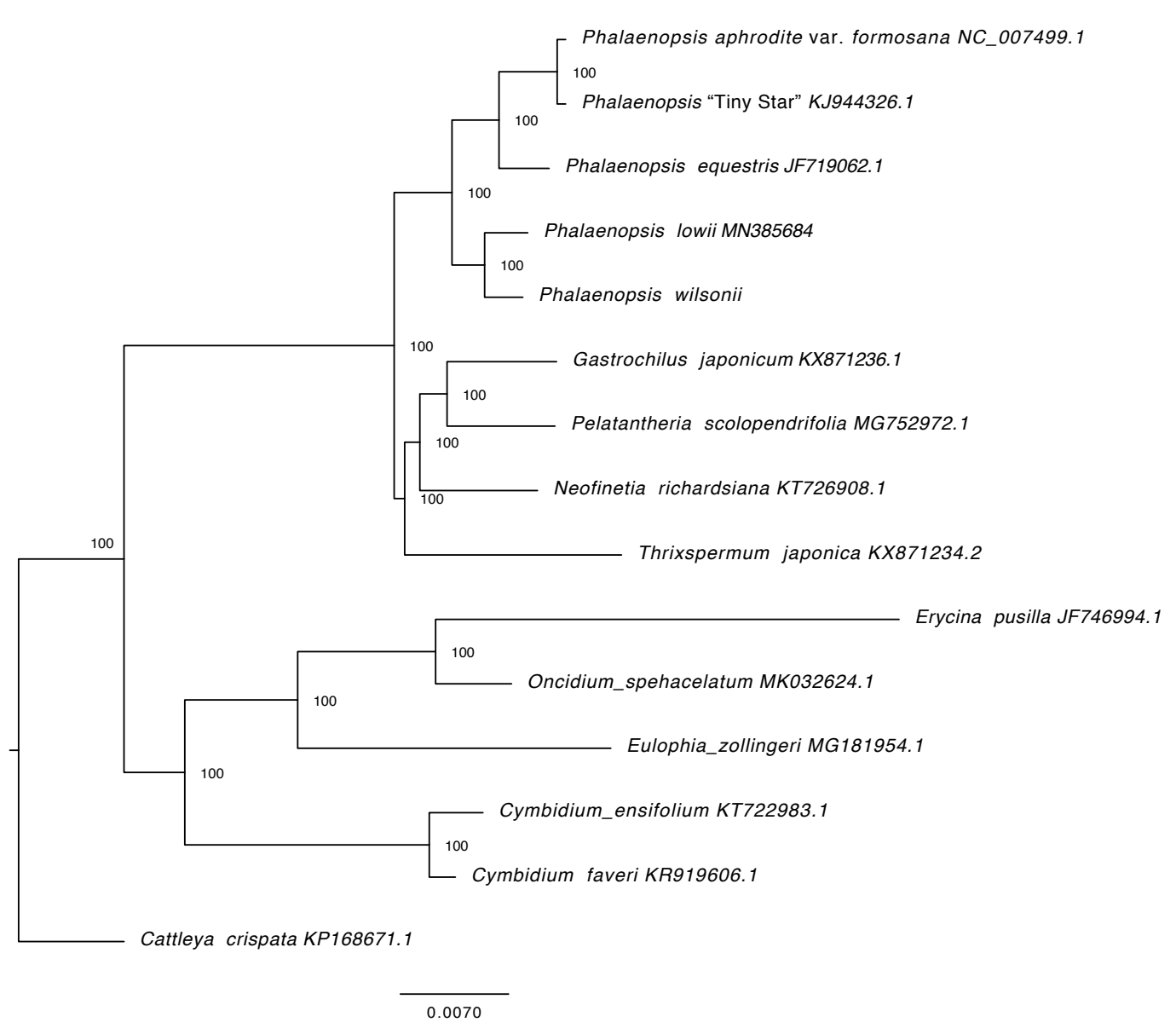

Figure 1. Maximum-likelihood tree reconstructed by RAxML based on complete chloroplast genome sequences from P. wilsonii, four Phalaenopsis species, and ten other orchids. Cattleya crispate was set as outgroup. Numbers on branches are bootstrap support values. 